[2] Fredericson $M$, Wolf $C$. lliotibial band syndrome in runners: innovations in treatment.Sports Med. 2005;35(5):451-9.

Disclosure of Interest: None declared

DOI: 10.1136/annrheumdis-2017-eular.3552

\section{SAT0604 AUTOLOGOUS BLOOD AND CORTICOSTEROID LOCAL INJECTION IN TREATMENT OF PLANTAR FASCIITIS (RANDOMIZED, CONTROLLED MULTICENTER CLINICAL TRIAL)}

A. Karimzadeh, S.A. Raeissadat, S. Erfani fam, L. Sedighipour. Physical Medicine \& Rehabilitation Research Center, Tehran, Iran, Islamic Republic Of

Background: Plantar fasciitis is the most common cause of heel pain. Local injection modalities are among treatment options in patients with resistant pain.

Objectives: The aim of the present study was to evaluate the effect of local autologous blood compared with corticosteroid local injection in treatment of plantar fasciitis.

Methods: In this randomized controlled multicenter study, 36 patients with chronic plantar fasciitis were recruited. Patients were allocated randomly into 3 treatment groups: local autologous blood, local corticosteroid injection and control groups receiving no injection. Patients were assessed with Visual Analogue Scale (VAS), Pressure Pain Threshold (PPT) and Plantar Fasciitis Pain/Disability Scale (PFPS) before treatment, 4 and 12 weeks post therapy.

Results: Variables of pain and function improved significantly in both corticosteroid and autologous blood groups compared to control group. At 4 weeks following treatment, patients in corticosteroid group had significantly lower levels of pain than patients in autologous blood and control groups (higher PPT level, lower PFPS and VAS). After 12 weeks of treatment both corticosteroid and autologous blood groups had lower average levels of pain than control group. The corticosteroid group showed an early sharp and then more gradual improvement in pain scores but autologous blood group had steady gradual drop in pain.

Conclusions: Autologous blood and corticosteroid local injection both can be considered as effective methods in the treatment of chronic plantar fasciitis. These treatments decrease pain and improve function significantly compared to control group.

Disclosure of Interest: None declared

DOI: 10.1136/annrheumdis-2017-eular.1771

\section{SAT0605 LOCAL PROGESTERONE INJECTION: NEW OPTION FOR MANAGEMENT OF CARPAL TUNNEL SYNDROME}

S.A. Raeissadat ${ }^{1}$, L. Sedighipour ${ }^{1}$, S. Shahraeeni ${ }^{2}$. ${ }^{1}$ Physical Medicine \& Rehabilitation Research Center; ${ }^{2}$ Physical medicine and rehabilitation research center, Tehran, Iran, Islamic Republic Of

Background: A number of studies, have demonstrated the neuroprotective effects of progesterone and its influence on the recovery after neural injury. Few studies investigated the efficacy of local progesterone in Carpal Tunnel Syndrome.

Objectives: The objective of this study was to compare the long term effects of progesterone versus corticosteroid local injections in patients with mild and moderate carpal tunnel syndrome.

Methods: In this randomized clinical trial, 78 hands with Carpal Tunnel Syndrome were assigned to two groups. Patients were treated with a single local injection of triamcinolone acetonide in one group and single local injection of hydroxy progesterone in the other group. Variables including pain (based on Visual Analogue Scale), symptom severity and functional status (based on Bostone/Levine symptom severity and functional status scale) and nerve conduction study were evaluated before and 6 months after the treatments.

Results: All outcome measures including pain, functional scales and electrophysiologic findings improved in both corticosteroid and progesterone groups and there were no meaningful differences between two groups regarding mentioned variables. However, functional outcome was significantly better in progesterone compared to corticosteroid group at 6 month follow up $(\mathrm{P}=0.04)$

Conclusions: This study demonstrated the efficacy of progesterone local injection in mild and moderate CTS at long term follow up. Furthermore, local progesterone can be superior to corticosteroid injection for relieving symptoms and improving functional and electrophysiologic findings at long term follow up.

Disclosure of Interest: None declared

DOI: 10.1136/annrheumdis-2017-eular.1774

\section{SAT0606 IS THE LEVEL OF PHYSICAL ACTIVITY AN IMPORTANT FACTOR FOR LOW BACK PAIN AMONG STUDENTS OF UNIVERSITY?}

V. Akduman ${ }^{1}$, E. Mete ${ }^{1}$, I. Demirbuken ${ }^{1}$, G. Ogren ${ }^{2} .{ }^{1}$ Physiotherapy and Rehabilitation; ${ }^{2}$ Nutrition and Dietetic, Marmara University, Istanbul, Turkey

Background: Back pain is a major global health problem, causing greater disability worldwide than any other condition. Regular physical activity is widely believed to have important health benefits, such as improving quality of life and mobility, and reducing disabilities. Conversely, lack of physical activity is considered a risk factor for increasing chronic diseases, functional dependence and mortality. Patients with low back pain (LBP) often report disability to perform daily activities. Also, decreased physical activity level can lead to low back pain. Objectives: The aim of the study was to determinewhether increasing the level of physical activity affects the low back pain or not in the population of univesity students.

Methods: The cross-sectional study included 350 students (181 females and 169 males) with a mean age of $19.8 \pm 1.9$ years. LBP was determined using the validated Oswestry Disability Index (ODI). ODI consist of ten items and are completed in reference to the patient's functional status "today". Physical activity level was evaluated by the short form of the International Physical Activity Questionnaire (IPAQ). IPAQ is a scale to be recorded at different levels of physical activity time in the last week. IPAQ is a scale to be recorded at different levels of physical activity time in the last week. Individuals whose score is lower than 600 MET are described as inactive (IPAQ 1), between 600-1500 MET (IPAQ 3) are described as minimal active and higher than 3000 MET (IPAQ 5)are described as active. For the statistical analyzing we used spearman correlation test.

Results: According to the short form of IPAQ, \%14.8 percent of students were found inactive, $\% 48.1$ percent of students were found minimal inactive and $\% 37.1$ percent of students were found active. The total score of Oswestry disability index was found $6,2 \pm 5,0$. There was a significant difference between the Oswestry disability index score of inactive, minimally active and active groups $(p=0,02)$. In addition to this, between the Oswestry disability index and total score of IPAQ was found a weak correlation in the negative direction $(p=0,01 ; r=-0,184)$. Between the IPAQwhich means vigorous intensity activity, and Oswestry disability index was found a weak correlation in the negative direction $(p=0,00 ; r=-0,190)$. And also between IPAQ3, which means modarate intensity activity, and Oswestry disability index was found a significant but weak correlation in the negative direction $(p=0,03 ; r=-0,157)$. Between Oswestry disability index and sedentary activity and walking was not found any significant correlation $(p>0.005)$.

Conclusions: According to our study, increasing the level of physical activity may reduce low back pain. Especially vigorous and modarate intensity activity can be effective in prevention from low back pain. For reducing of low back pain, sedentary activity and walking did not have any significant effect in our study. Therefore, the level of physical activity should be increased at the young age to prevent of low back pain which is a major health problem.

References:

[1] Saglam, Melda, et al. International physical activity questionnaire: reliability and validity of the Turkish version. Perceptual and motor skills 111.1 (2010): 278-284.

[2] Fairbank, Jeremy CT, and Paul B. Pynsent. The Oswestry disability index. Spine 25.22 (2000): 2940-2953.

Disclosure of Interest: None declared

DOI: 10.1136/annrheumdis-2017-eular.4953

\section{SAT0607 DISCOVERY OF A SMALL MOLECULE INHIBITOR OF THE WNT PATHWAY (SM04755) AS A POTENTIAL TOPICAL TREATMENT FOR TENDINOPATHY}

V. Deshmukh, T. Seo, M. Ibanez, J. Stewart, B. Hofilena, Y. Yazici. Samumed, LLC, San Diego, CA, United States

Background: Tendinopathy is an inflammatory and degenerative disorder caused by injuries or overuse. It can progress to a chronic condition with failed healing, tendon fibrosis and micro-tears that lead to pain and sometimes rupture. Current therapeutic options focus mainly on pain relief rather than treatment of underlying disease. The Wnt pathway is upregulated in tendinopathy and has an important role in inflammation, fibrosis and tenocyte differentiation.

Objectives: SM04755, a novel, topical Wnt pathway inhibitor, was evaluated in preclinical studies to determine its potential to inhibit inflammation, reduce fibrosis and increase tenocyte differentiation, thereby promoting tendon healing.

Methods: Anti-inflammatory activity was measured by TNF- $\alpha$ and IL- 6 secretion using ELISA in lipopolysaccharides (LPS) or anti-CD3/anti-CD28 stimulated peripheral blood mononuclear cells (PBMCs). Differentiation of human mesenchymal stem cells (hMSCs) and rat tendon derived stem cells (rTDSCs) into tenocytes was measured by high-content imaging for tenocyte markers scleraxis A (SCXA), tenomodulin and tenascin C. Pharmacokinetics were evaluated following topical application in rats. In vivo efficacy of SM04755 was evaluated in a single injection, collagenase-induced acute rodent tendinopathy model and a chronic, multiple injection, failed healing model, by scoring histological indicators of tendon health. Inflammation was measured by chemokine ligand 1 (CXCL1) levels in plasma by ELISA and pro-inflammatory markers (IL-6, TNF- $\alpha$, IL-1 $\beta$, IFN- $\gamma$, IL- 8 ) in the tendon by qPCR. Tendon regeneration and healing were evaluated by qPCR based gene expression of tenocyte differentiation markers SCXA, tenomodulin and tenascin C, Type I/Type III collagen ratio and polarized light microscopy using Sirus Red staining. Pain in the rodent model was evaluated by measuring weight distribution with an incapacitance meter.

Results: SM04755 potently inhibited cytokine secretion in LPS and antiCD3/anti-CD28 stimulated PBMCs $\left(E_{50}=500 \mathrm{nM}\right)$. SM04755 induced expression of tenocyte markers in differentiated hMSCs and rTDSCs $\left(E_{50}=200 \mathrm{nM}\right)$. A single topical application of SM04755 resulted in tendon concentrations $>\mathrm{EC}_{50}$ for up to $24 \mathrm{hrs}$, with minimal systemic exposure or toxicity. In both the acute and failed healing tendinopathy models, SM04755 $(10 \mathrm{mg} / \mathrm{ml})$ treatment improved tendon morphology (Figure A), significantly increased mean tendon health 
score $(p<0.01)$, decreased plasma levels of CXCL1 $(p<0.05)$ and reduced gene expression of pro-inflammatory markers (IL-6, TNF-a, IL-1b, INF-g, IL-8; $\mathrm{p}<0.05)$ compared to vehicle. SM04755 treatment promoted tendon regeneration measured as increased expression of tenocyte markers $(p<0.05)$, increased Type I/Type III collagen ratio (Figure $B ; p<0.01$ ) and Sirus Red stained collagen fibers in tendon compared to vehicle. SM04755 treatment increased \% total weight bearing on the affected limb $(p<0.01)$, at multiple time points (Figure $C)$, indicating reduced pain in the rodent model.

Figure. SM04755 inhibited inflammation, promoted tendon healing and reduced pain in a rat collagenase-induced tendinopathy model
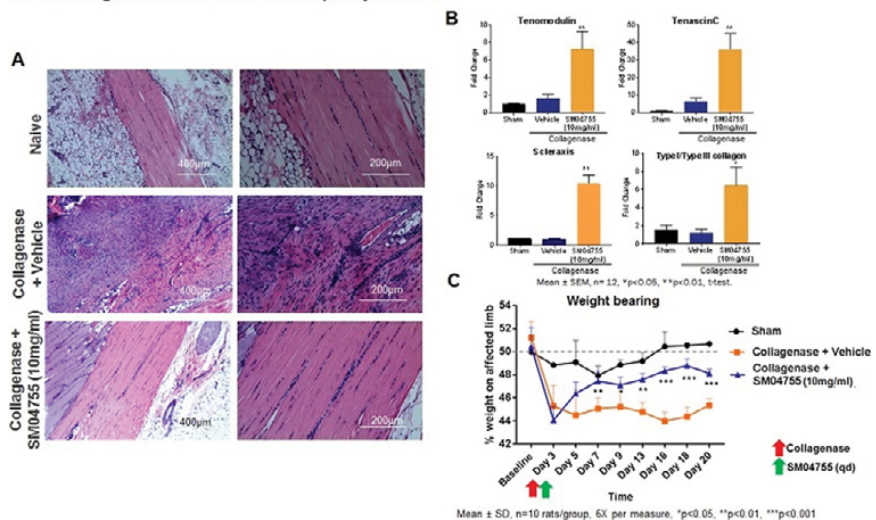

Conclusions: Topical SM04755, a Wnt pathway inhibitor, reduced inflammation, promoted tendon regeneration and healing, and reduced pain compared to vehicle in rodent tendinopathy models. SM04755 is a potential treatment for tendinopathy. Clinical studies are in progress.

Disclosure of Interest: V. Deshmukh Shareholder of: Samumed, LLC, Employee of: Samumed, LLC, T. Seo Shareholder of: Samumed, LLC, Employee of: Samumed, LLC, M. Ibanez Shareholder of: Samumed, LLC, Employee of: Samumed, LLC, J. Stewart Shareholder of: Samumed, LLC, Employee of: Samumed, LLC, B. Hofilena Shareholder of: Samumed, LLC, Employee of: Samumed, LLC, Y. Yazici Shareholder of: Samumed, LLC, Employee of: Samumed, LLC DOI: 10.1136/annrheumdis-2017-eular.6427

\section{SAT0608 RELATION BETWEEN SCAPULAR MUSCLE ENDURANCE, MUSCLE STRENGTH, PAIN AND FUNCTION IN PATIENTS WITH ROTATOR CUFF LESION}

U. Sozlu, S. Basar, U. Kanatli, Z.B. Alkan. Gazi University, Ankara, Turkey

Background: Rotator cuff lesion (RCL) is an term that encompasses a spectrum of shoulder conditions including; subacromial impingement syndrome (SIS), partial and full thickness rotator cuff tears (RCT) and calcific tendinitis. Symptoms include pain, limited motion, muscle weakness and functional disability.

Objectives: To investigate the relationship between scapular muscle endurance (SME), muscle strength, pain and function in patients with shoulder RCL.

Methods: This study was conducted on 53 patients in shoulder RCL and 23 healthy controls. SIS was present in 25 patients $(14$ males, 11 females; mean age $41 \pm 9$ years) and partial RCT in 28 patients ( 16 females, 12 males; mean age was $54 \pm 5$ years) in the study group. In the control group, there were 12 females and 11 males and the mean age was $48 \pm 10$ years. SME was measured as isometric based on the exercise developed by Sahrmann. Strength of the shoulder and scapular muscles were evaluated with digital manuel muscle dynamometer. Activity, night and rest pain levels were evaluated by visual analog scale. Functional activity status was assessed by the Functional Impairment Test-Head, and Neck/Shoulder/Arm (FIT-HaNSA).

Results: Statistically significant relationship was found positively in varying grades between SME and serratus anterior, middle trapezius, shoulder flexor, extensor, abductor, supraspinatus, pectoralis majör strength in partial RCT group and between lower trapezius muscle strength and SME in addition to these muscles in SIS group $(p<0.05)$. Statistically significant relationship at varying degrees was found between SME and all muscles excluding shoulder internal, external rotators in control group $(p<0.05)$. In SIS and partial RCT groups, negative, moderate and statistically significant relationship was found between SME and activity pain $(p<0.05)$. In SIS and partial RCT groups significant correlation was not observed between SME and rest, night pain levels $(p>0.05)$. In SIS and partial RCT groups, positive, excellent and statistically significant relationship was detected between SME and FIT-HaNSA $(p<0.05)$. In control group, positive, moderate, again statistically significant relationship was detected $(p<0.05)$.

Conclusions: Muscle strength may be reduced, upper extremity function may worsen and activity pain may increase in patients with reduced SME. It was concluded that shaping of exercise programs of muscle groups related to SME in rehabilitation studies and SME should be increased in patients with SIS and partial RCT in order to reduce shoulder pain and improve its functionality.

References:

[1] Cofield, R. H. (1985). Current concepts review rotator cuff disease of the shoulder. Journal of Bone and Joint Surgery. American volume, 67(5), 974-979.

[2] Neer, C. S. (1993). Impingement lesions. Clinical Orthopedia, 173, 71-7.

[3] Edmondston, S. J., Wallumrød, M. E., MacLéid, F., Kvamme, L. S., Joebges, S. and Brabham, G. C. (2008). Reliability of isometric muscle endurance tests in subjects with postural neck pain. Journal of Manipulative and Physiological Therapeutics, 31(5), 348-354.

[4] MacDermid, J. C., Ghobrial, M., Quirion, K. B., St-Amour, M., Tsui, T., Humphreys, D., McCluskie, J., Shewayhat, E. and Galea, V. (2007). Validation of a new test that assesses functional performance of the upper extremity and neck (FIT-HaNSA) in patients with shoulder pathology. BMC Musculoskeletal Disorders, 8(1), 42.

Disclosure of Interest: None declared

DOI: 10.1136/annrheumdis-2017-eular.6310

\section{SATURDAY, 17 JUNE 2017 Diagnostics and imaging procedures}

\section{SAT0609 GIANT CELL ARTERITIS: ATHEROSCLEROSIS AS PITFALL IN THE HALO SIGN}

E. De Miguel ${ }^{1}$, L.M. Beltran ${ }^{2}$, F. Deodati ${ }^{2}$, I. Monjo ${ }^{1}$, W.A. Schmidt ${ }^{3}$,

J. García-Puig ${ }^{2} .{ }^{1}$ Rheumatology; ${ }^{2}$ Internal Medicine, Hospital Universitario la Paz, Madrid, Spain; ${ }^{3}$ Rheumatology, Immanuel Krankenhaus, Berlin, Germany

Background: The halo sign (defined as an homogenous, hypoechoic wall thickening, well delineated towards the luminal side, visible in longitudinal and transverse planes). is the more accurate ultrasonographic (US) finding for the diagnosis of Giant Cell Arteritis (GCA). False-positive halos are seen in malignant and infectious diseases. The new US high frequencies probes make possible not only to see the halo sign but also measure the increase of the intima-mediathickness (IMT). Moreover, the US appearance of the IMT in atherosclerotic carotid artery may be hypoechoic as the halo sign.

Objectives: The aim of this study was to explore if the increase of the atherosclerotic IMT can mimic the halo sign in temporal arteries (TA) and to produce false positives in the diagnosis of GCA.

Methods: Consecutive non selected patients, $>50$ years-old with high vascular risk according to European Guidelines on Cardiovascular Disease Prevention, and without signs or symptoms of GCA, were included.

Ultrasonography of carotid artery: Carotid ultrasound examinations were performed on a Mylab Seven (Esaote Medical Systems, Italy) with a 4-13 MHz linear-array. The system employed dedicated software radiofrequency-tracking technology to obtain IMT $\left(\mathrm{QIMT}^{\circledR}\right)$.

Ultrasonography of temporal superficial artery: A color Doppler ultrasound (CDU) and grey scale measure of the IMT/halo sign in the branches of both TA was performed by a second experienced sonographer. A Mylab Twice equipment (Esaote, Geneve, Italy) was used, with a $22 \mathrm{MHz}$ frequency for grey scale and a $12.5 \mathrm{MHz}$ for CDU, with a color gain of 51 and a PRF of $2 \mathrm{kHz}$. The sonographer was blind to the clinical data and carotid ultrasound IMT measures. Examination videos were stored for reliability and an intra-reader was performed months after the examination.

Statistical analysis: Descriptive frequencies, Student's $t$-test, Cronbach's alpha and Spearman correlations was used.

Results: Forty patients were studied, 28 men $(70 \%)$, with a mean age of $70,6 \pm 6,9$ years. Three patients were active smoker and 27 ex-smokers. Arterial hypertension was present in $39(97.5 \%)$, dyslipidemia in $34(85 \%)$ and diabetes in $19(47.5 \%)$. The mean erythrocyte sedimentation rate was $13.6 \pm 11.0$. Eighty carotids were studied, 50 had plaques and 30 did not with a IMT ranged from 0.528 to $1.480 \mathrm{~mm}$. The mean values of TA related with the carotid IMT are presented in the table: the increase in the carotid IMT is associated with an increase in the IMT of the TA with a weak Spearman correlation (parietal branches $0.282 p=0.012$ and frontal branches $0.228 p=0.048$ )

Patients with a final GCA diagnosis $(n=22)$

\begin{tabular}{lcccc}
\hline Carotid IMT mm & \multicolumn{4}{c}{ Branches of Temporal arteries parietal and frontal } \\
\cline { 2 - 5 } & $\mathrm{n}$ & Mean Halo/IMT mm & SD & Min-Max \\
\hline$\leq 0.7$ & 12 & 0.247 & \pm 0.043 & $0.16-0.29$ \\
$\leq 0.9$ & 46 & 0.254 & \pm 0.037 & $0.18-0.33$ \\
$\leq 1$ & 42 & 0.269 & \pm 0.034 & $0.18-0.36$ \\
$\leq 1.2$ & 50 & 0.282 & \pm 0.052 & $0.17-0.45$ \\
$\geq 1.2$ & 10 & 0.284 & \pm 0.067 & $0.19-0.40$ \\
\hline
\end{tabular}

From reliability a Cronbach's alpha of 0.900 and 0.876 were achieved for parietal and frontal branches respectively.

Some patients had a TA IMT that can be interpreted as halo sign: $18(45 \%)$ patients and $33(20.6 \%)$ TA branches if we choose a cutoff $>0.30 \mathrm{~mm}$ of IMT/halo sign; $4(10 \%)$ patients and $7(4.4 \%)$ TA branches if the cutoff were $>0.34 \mathrm{~mm}$.

Conclusions: The atherosclerotic disease can produce false-positive GCA diagnosis. Carotid IMT $>0.9 \mathrm{~mm}$ is associated with halo sign in TA. The IMT cutoff value for the diagnosis of GCA should be established.

Disclosure of Interest: None declared

DOI: 10.1136/annrheumdis-2017-eular.1461 